\title{
Nanospring behaviour of ankyrin repeats
}

\author{
Gwangrog Lee ${ }^{1}$, Khadar Abdi ${ }^{2}$, Yong Jiang ${ }^{1}$, Peter Michaely ${ }^{3}$, Vann Bennett ${ }^{2}$ \& Piotr E. Marszalek ${ }^{1}$
}

Ankyrin repeats are an amino-acid motif believed to function in protein recognition; they are present in tandem copies in diverse proteins in nearly all phyla ${ }^{1}$. Ankyrin repeats contain antiparallel $\alpha$-helices that can stack to form a superhelical spiral ${ }^{2}$. Visual inspection of the extrapolated structure of 24 ankyrin-R repeats ${ }^{2}$ indicates the possibility of spring-like behaviour of the putative superhelix. Moreover, stacks of 17-29 ankyrin repeats in the cytoplasmic domains of transient receptor potential (TRP) channels have been identified as candidates for a spring that gates mechanoreceptors in hair cells as well as in Drosophila bristles $^{3-5}$. Here we report that tandem ankyrin repeats exhibit tertiary-structure-based elasticity and behave as a linear and fully reversible spring in single-molecule measurements by atomic force microscopy. We also observe an unexpected ability of unfolded repeats to generate force during refolding, and report the first direct measurement of the refolding force of a protein domain. Thus, we show that one of the most common aminoacid motifs has spring properties that could be important in mechanotransduction and in the design of nanodevices.

The atomic structure of 12 ankyrin-R repeats suggests that ankyrin stacks composed of $n \geq 24$ repeats should form a full superhelical turn with putative spring properties ${ }^{2,3}$. We used an atomic force microscope (AFM) to identify individual stacks of 24 ankyrin-B repeats (Supplementary Fig. S2) and found that they do indeed have a hook-like shape ${ }^{2}$ with the molecules' end-to-end distance closely matching the $\sim 12 \mathrm{~nm}$ determined for the extrapolated structure ${ }^{2}$ (Fig. 1a). Thus, the AFM images strongly suggest that the engineered protein, bearing at its terminus a glutathione $S$-transferase (GST) module, is correctly folded and does not aggregate. These conclusions are further supported by circular dichroism and hydrodynamic measurements (Supplementary Table 1 and Supplementary Fig. S1).

For elasticity measurements, heptahistidine-tagged polypeptides containing 24 ankyrin-B repeats with or without GST, or 12 repeats with GST, were immobilized on a glass surface bearing the metal chelate $N$-nitrilotriacetic acid (NTA) ${ }^{6,7}$ (Fig. 1a). Molecules were stretched vertically, in solution, by the AFM cantilever, and their length and tension were measured with subnanometre and $\sim 10 \mathrm{pN}$ precision $^{8-10}$. Most trials revealed complex force-extension profiles with irregularly spaced force peaks typical of multiple molecules (Supplementary Fig. S4a). However, $\sim 5 \%$ of the force-extension curves had simple and consistent features that, we argue, represent
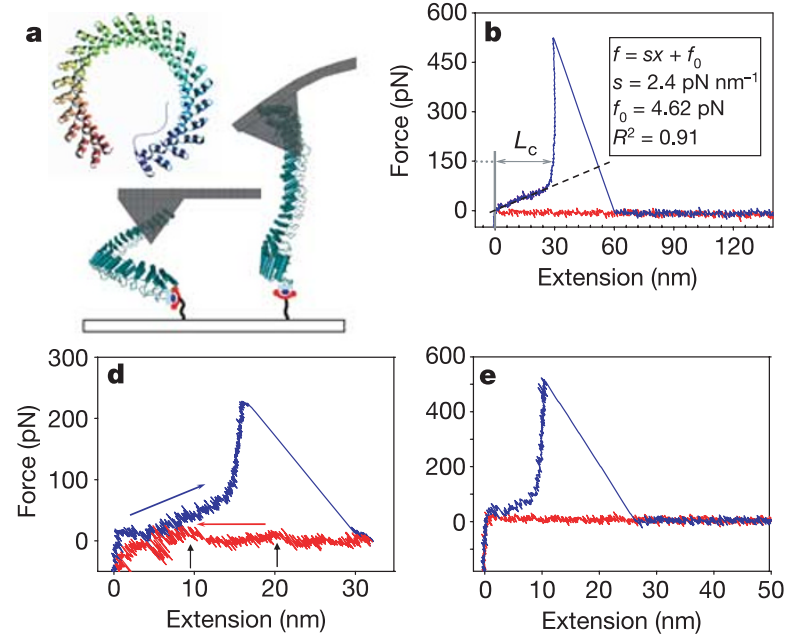
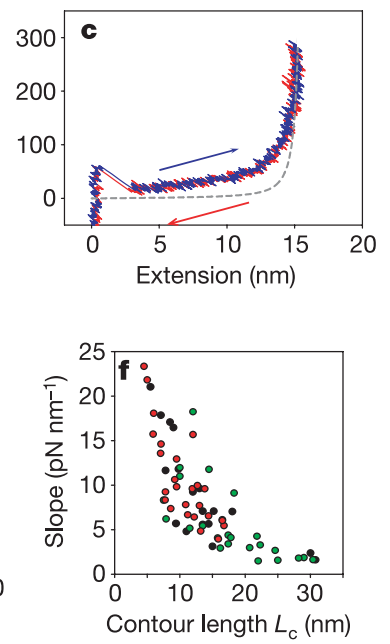

Figure 1 Atomic force microscopy measurements reveal the linear elasticity of ankyrin-B repeats. a, The extrapolated structure of 24 ankyrin$R$ repeats ${ }^{2}$ and a diagram of the elasticity measurement on a His-tagged ankyrin fragment bound to NTA (red handles) and stretched with the AFM cantilever. b-e, Force-extension curves of individual ankyrins: 24 repeats with GST (b-d); 24 repeats with no GST (e). In b, the molecule detached from the AFM at the stretching force peak (blue); in c, the molecule's attachment was preserved after stretching (blue trace) and the molecule was subsequently relaxed (red trace); in $\mathbf{d}$, the molecule unfolded at the stretching force peak (blue) but remained attached to the AFM. The inset in b shows how ankyrin spring constant $s$ was determined by fitting the straight-line equation to the data: $f$, force; $f_{0}$, force offset; $x$, extension; $R^{2}$, coefficient of determination; $L_{c}$, contour length (determined as the extension at $150 \mathrm{pN}$ ). In $\mathbf{d}$, the relaxing trace (red) after the unfolding of ankyrin that occurred at the stretching force peak (blue) revealed small force peaks (arrows) indicating the refolding of individual repeats (see also Supplementary Fig. S6). f, Ankyrin spring constant decreases with the increasing length of the stretched fragment. Black dots, 24 repeats with GST; green dots, 24 repeats with no GST; red dots, 12 repeats with GST. 
the elasticity of single ankyrin stacks. Figure 1b-e shows 4 of 77 similar recordings. Interestingly, at forces of $100 \mathrm{pN}$ or less, all of these curves display a linear region where force is directly proportional to extension. The length $L_{\mathrm{c}}$ of the stretched segment (Fig. 1b, inset) varied between $\sim 5$ and $\sim 30 \mathrm{~nm}$, which is consistent with random adsorption of the AFM tip along the ankyrin polypeptide. Provided that stretching was stopped before unfolding, the extension was fully reversible (compare the red and blue traces in Fig. 1c). In other cases where the molecules detached (Fig. 1b, e) or were unfolded (Fig. 1d), extension was not reversible.

The fact that Fig. 1b, d, e displays a single force peak strongly indicates that these recordings were obtained on single molecules. Note also that the relaxing trace in Fig. 1d (red curve) shows small refolding force peaks (arrows) generated by individual ankyrin repeats (see below and Supplementary Fig. S6). Moreover, the force curves shown in Fig. 2b, c show an initial linear phase followed by rupture of the repeat stack and unfolding of individual repeats (see below). Together, these traces provide direct evidence that the unusual linear elasticity captured in our AFM recordings on ankyrin do indeed represent the properties of individual molecules.

Unlike most modular proteins studied by atomic force microscopy, including titin $^{8,10-13}$, tenascin ${ }^{9}$, fibronectin ${ }^{14}$, ubiquitin ${ }^{15}$, spectrin $^{16}$ and filamin ${ }^{17}$, whose elasticity typically follows a highly nonlinear entropic behaviour exemplified by the worm-like chain (WLC) model $^{18}$ (Fig. 1c, dashed line), ankyrin is a hookean or linear spring for which the tension is proportional to the extension. To behave similarly to ankyrin, a WLC polymer would need an unphysical persistence length of less than $1 \AA$.

Elastic properties of ankyrin repeats have been proposed on the basis of their coil-like shape $e^{3,19,20}$. Recent steered molecular dynamics (SMD) simulations also predict that ankyrin-R repeats stretch reversibly by straightening the superhelical turn, when subjected to forces of $\sim 25-100 \mathrm{pN}$. These simulations predicted that the spring constant of ankyrin- $\mathrm{R}$ is $4.1-4.7 \mathrm{pN} \mathrm{nm}^{-1}$ for 24 repeats and increases to $16.4 \mathrm{pN} \mathrm{nm}^{-1}$ for 12 repeats $^{5}$, indicating that the stiffness of the stack increases with a decreasing number of repeats. We plotted the spring constants of various ankyrin constructs as a function of $L_{c}$ (Fig. 1f) and found that longer ankyrin stacks are on average more compliant than shorter ones, which is consistent with expectations of a hookean spring and the SMD simulations ${ }^{5}$. Moreover, the GST-tagged protein does not contribute to the spring constant. The range of spring constants is $1.5-21 \mathrm{pN} \mathrm{nm}^{-1}$ for 24 ankyrin-B repeats and $4.0-23 \mathrm{pN} \mathrm{nm}^{-1}$ for the 12-repeat construct. The spring constant measured for the longest fragments (extensions: $25-30 \mathrm{~nm}$; Fig. 1f) presumably containing all 24 ankyrin repeats, is $1.87 \pm 0.31 \mathrm{pN} \mathrm{nm}^{-1}$ (mean \pm s.d., $n=5$ ). For comparison, the estimate based on the SMD calculation gives $4.4 \mathrm{pN} \mathrm{nm}^{-1}$ (ref. 5) and the spring constant of a spring that gates mechanoreceptors in hair cells was predicted to be $0.5 \mathrm{pN} \mathrm{nm}^{-1}$ (ref. 21).

Ankyrin-B repeats extend reversibly in multiple stretch-relaxation cycles with no signs of hysteresis or wear over a large range of applied forces exceeding $100 \mathrm{pN}$ (Fig. 1c). However, these stacks do unfold at relatively high forces ranging from 200 to $1,250 \mathrm{pN}(365 \pm 202 \mathrm{pN}$; mean \pm s.d., $n=32$; see, for example, Figs $1 \mathrm{~d}$ and 2; Supplementary Fig. S9e). Figure 2 describes the mechanical unfolding of ankyrin stacks in detail. The first force peak, labelled A in Fig. 2a, is a rupture of a nonspecific adhesive bond between the AFM tip and the substrate. The next force peak of $310 \mathrm{pN}$ reports the breakdown of the molecule and not its detachment from the tip (stage 1, peak labelled $\mathrm{U}$ ) as demonstrated by the rest of the force curve, which captured the WLC-like elasticity (stage 2$)^{22}$. From the length of the polypeptide chain liberated after the breakdown (U) we estimate that six repeats were unfolded simultaneously and subsequently stretched. Interestingly, further extension revealed a sawtooth pattern of six regularly spaced force peaks, each separated by $12.4 \mathrm{~nm}$ (on the basis of the WLC fits). This distance matches the length of an ankyrin repeat (33 residues $\times 0.36 \mathrm{~nm}$ per residue), indicating that
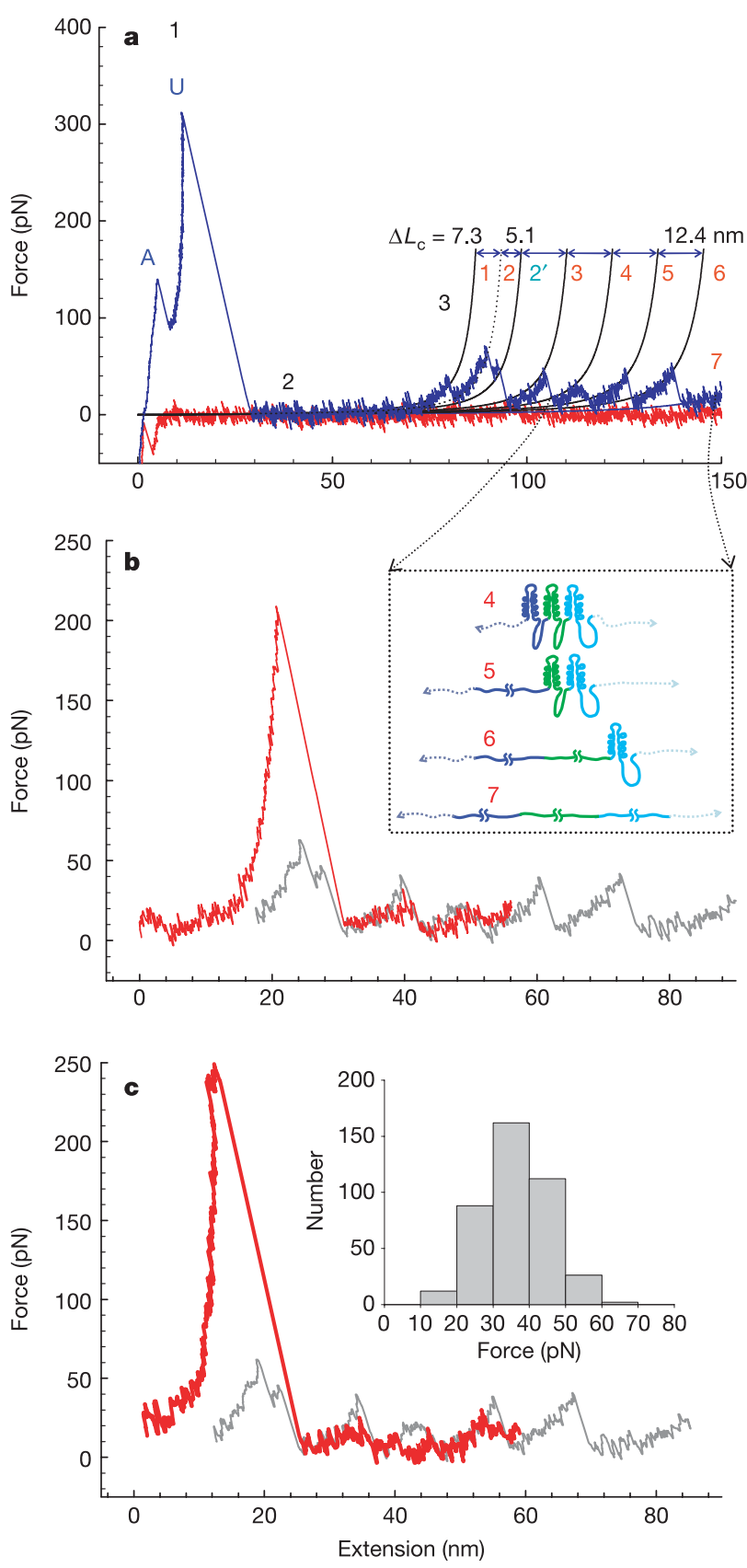

Figure $2 \mid$ Atomic force microscopy captures the mechanical breakdown of the ankyrin stack and the unfolding of individual repeats. a, The first force peak in the force-extension curve at $\sim 150 \mathrm{pN}$ (A) represents a rupture of a nonspecific adhesion bond. At $310 \mathrm{pN}$ the stack breaks down $(1, \mathrm{U})$; and about six repeats unravel cooperatively and are stretched in a WLC manner (curve labelled 2). At point 3 there is a sequential unfolding of the remaining repeats. Thin solid lines are the WLC fits to the data with a persistence length $p$ of $2 \mathrm{~nm}$ and the contour length increment $\Delta L_{\mathrm{c}}$ of $12.4 \mathrm{~nm}$. Note that the second peak is actually composed of two peaks $\left(2\right.$ and $\left.2^{\prime}\right)$ separated from the first peak by 7.3 and $12.4 \mathrm{~nm}$, respectively. b, A force-extension curve of another ankyrin fragment reveals in a single pull its linear elasticity, the breakdown of the stack at $\sim 240 \mathrm{pN}$, and the unfolding of individual repeats (red trace). Inset, our interpretation of the events occurring in stage 3 of a. c, As in b, but obtained on a different ankyrin fragment. The inset shows a histogram of unfolding forces of individual repeats. The grey trace in $\mathbf{b}$ and $\mathbf{c}$ comes from the force-extension curve shown in a.

the force peaks might correspond to the sequential unfolding of individual ankyrin repeats. The force that is necessary to unfold a single ankyrin repeat (Figs 2 and 3; Supplementary Figs S9 and S10) is $37 \pm 9 \mathrm{pN}$ (mean \pm s.d., $n=404$ force peaks; Fig. 2c, inset), which 

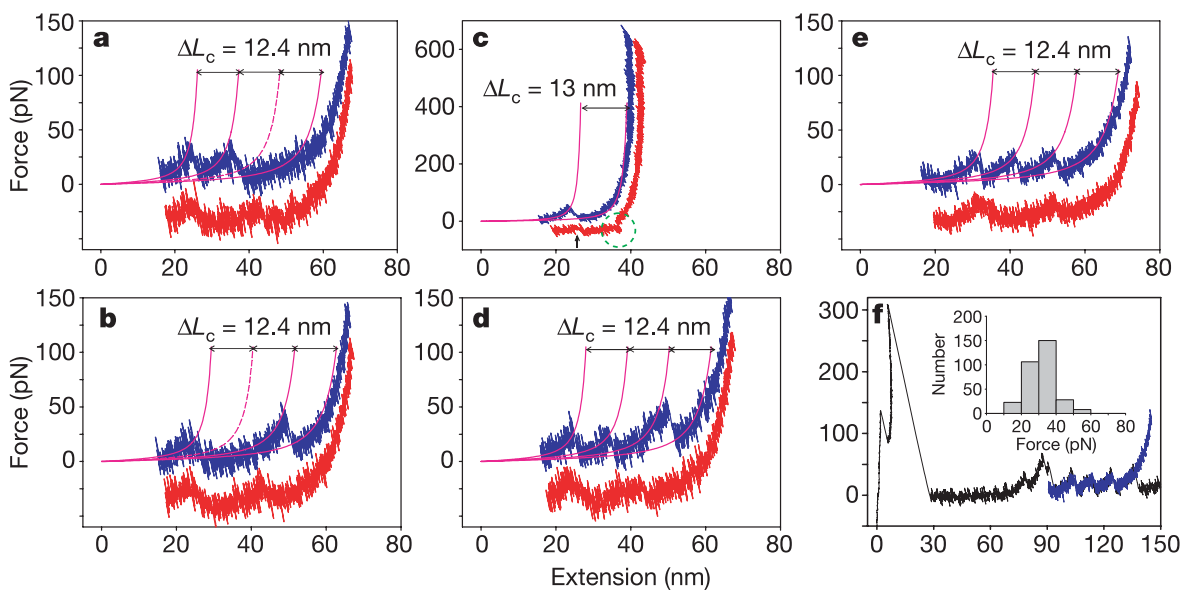

Figure 3 Atomic force microscopy directly measures the refolding forces of ankyrin repeats. a-e, A sequence of stretch (blue traces) and release (red traces) measurements of a single partly unfolded ankyrin fragment (compare with Supplementary Fig. S7). Thin lines are the WLC fits with $p=1 \mathrm{~nm}$ and $\Delta L_{\mathrm{c}}=12.4 \mathrm{~nm}$. The relaxing traces are offset vertically for clarity. a, Cycle 4. b, Cycle 5. c, Cycle 6: two of the three previously unfolded repeats reattached to the stack and only a single repeat unfolded. Green dotted circle, instability of the refolded stack captured in the relaxing trace. d, Cycle 7: the stretching trace reveals that the two repeats that had reattached to the stack now detached again and all three repeats unfolded sequentially, then refolded. e, As in d, but obtained after 26 more stretch-release cycles. $\mathbf{f}$, The unfolding trace of e superimposes perfectly on the template trace of Fig. 2a. The inset shows a histogram of the refolding forces of individual repeats. is similar to the unfolding force $(25-35 \mathrm{pN})$ of individual spectrin domains ${ }^{16}$.

Figure 2b, c shows two additional examples of the forced unfolding of different ankyrin stacks, each captured in a single measurement. They clearly reveal the linear elasticity of the stack followed by its breakdown (high force peak), which in turn is followed by an unravelling of individual repeats (small force peaks). Significantly, these small force peaks overlap well with the small force peaks captured in Fig. 2a (shown in Fig. 2b, c as a grey trace), strongly indicating that all these force peaks represent the unfolding of individual repeats (see Fig. 2b, inset).

A remarkable feature of ankyrin repeats is their ability to refold in a process that reveals significant refolding forces (Fig. 1d). We further investigated refolding in a sequence of 33 consecutive stretch-release recordings obtained on a single ankyrin fragment (Fig. 3; see also Supplementary Figs S7-S9). The recordings in Fig. 3a-e were obtained on ankyrin that had already been partly unfolded and prestretched by $17 \mathrm{~nm}$ (Supplementary Fig. S7). Figure 3a (blue trace) revealed two small force peaks of $32 \mathrm{pN}$, separated by $\sim 12 \mathrm{~nm}$, similar to those in Fig. 2a, followed by the force curve, which was separated from the second peak by $\sim 25 \mathrm{~nm}$. This result indicates that the unfolding of a single ankyrin repeat might have been followed by the simultaneous unfolding of two repeats. The relaxing trace in Fig. 3a (red curve) also shows two force peaks of $27 \mathrm{pN}$ and their spacing suggests that they report the refolding of a single repeat that was followed by the refolding of the two remaining repeats. The next cycle (Fig. 3b) shows a similar pattern, except that now two repeats unfolded first and a single repeat unfolded later. However, the refolding order was the same as in the previous measurement. This observation may reflect some strict refolding order similar to p16 ankyrin repeats, in which carboxy-terminal repeats fold before the amino-terminal repeats ${ }^{23}$.

The sixth stretching cycle on the same molecule (Fig. 3c), which was previously stretched to $66 \mathrm{~nm}$, now yielded a maximum extension of $40 \mathrm{~nm}$. This result indicates that two of the three repeats that refolded in the event captured in Fig. 3b (red trace) must also have reattached to the core of the stack and did not unfold in the subsequent pull (see also Supplementary Figs S8 and S9). The fact that the reconstructed stack can sustain forces in excess of $600 \mathrm{pN}$ (Fig. 3c) indicates enormous cohesive forces between neighbouring ankyrin repeats in the stack. This observation also suggests that the (small) force peaks in Fig. 3a, b probably correspond to the mechanical unfolding of the repeats that detached earlier from the stack and remained separated from it even after refolding. Taken together, these results are consistent with observations of very favourable interfacial interactions between the nearest repeats in the stacks of ankyrin repeats ${ }^{24}$. The next and subsequent stretching cycles (Fig. 3d, e) revealed three evenly spaced force peaks and two refolding force peaks, indicating that the repeats that had reattached to the stack detached again and were sequentially unfolded. The unfolding patterns shown in Fig. 3 overlap perfectly with the sawtooth pattern of Fig. 2a (Fig. 3f), indicating that the refolding of repeats occurs with high fidelity.

Altogether, we observed a similar refolding behaviour of ankyrin repeats in 75 separate experiments performed on different molecules on different days, using different AFM instruments and cantilevers. In more than 100 separate observations (including different recordings on the same molecule) we recorded major refolding of the stack as shown in Fig. 3c (see also Supplementary Fig. S9d, e). The average refolding force was $32 \pm 8 \mathrm{pN}$ (mean \pm s.d., $n=315$ force peaks; Fig. 3f, inset). Although refolding of proteins under force has been observed previously ${ }^{15,25,26}$, here we have made direct measurements on single molecules that capture the magnitude of the refolding force of a protein domain.

Ankyrins interact directly with cytoplasmic domains of a variety of membrane transporters and cell adhesion molecules, and couple these proteins to the spectrin-based membrane skeleton ${ }^{27}$. We propose that the linear elasticity of 24 ankyrin-B repeats shown here might have a role in modulating activity of ankyrin-associated transporters in response to mechanical strain and/or in generating tension in the plane of the membrane bilayer. More generally, the unusually strong tendency of ankyrin repeats to refold may be of particular significance to proteins with four to six ankyrin repeats. Finally, the mechanical properties of ankyrin repeats shown here make them ideal candidates as the building blocks of bio-inspired springy nanostructures and nanomaterials with an inherent ability to self-repair.

\section{METHODS}

Purification and characterization of ankyrin-B polypeptides. These are described in Supplementary Information.

Immobilization of ankyrin molecules. Glass coverslips were functionalized with the metal chelate NTA as described in refs $6,7 . \mathrm{NiCl}_{2}$ solution $(50 \mu \mathrm{l}$, $50 \mathrm{mM}$ ) was loaded onto the NTA-functionalized glass for $5 \mathrm{~min}$ to achieve 
chelation, and the glass was rinsed gently with solution composed of $20 \mathrm{mM}$ phosphate buffer supplemented with $300 \mathrm{mM} \mathrm{NaCl} ; 40 \mu \mathrm{l}$ of solution containing heptahistidine-tagged ankyrin molecules at a concentration of $2-10 \mu \mathrm{g} \mathrm{ml}^{-1}$ was deposited on a glass coverslip and incubated at $20-25^{\circ} \mathrm{C}$ for $20 \mathrm{~min}$.

AFM stretching measurements. All pulling measurements were made with custom-built AFM instruments ${ }^{9}$ equipped with an AFM detector head (Veeco Metrology group) and high-resolution piezoelectric stages (Physik Instrumente) equipped with position sensors (a vertical resolution $0.1 \mathrm{~nm}$ ). The spring constant of each MLCT-AUHW micro-cantilever (Veeco) was calibrated by using the energy equipartition theorem as described in ref. 28. Molecules were picked up for stretching measurements by gently touching the substrate with the AFM tip, exploiting a nonspecific adsorption of ankyrin to the tip. Forceextension measurements were performed at pulling speeds of between 0.012 and $0.2 \mathrm{~nm} \mathrm{~ms}^{-1}$, in solution and at room temperature.

\section{Received 28 August; accepted 15 November 2005.}

Published online 15 January 2006.

1. Mosavi, L. K., Cammett, T. J., Desrosiers, T. J. \& Peng, Z. Y. The ankyrin repeat as molecular architecture for protein recognition. Protein Sci. 13, 1435-1448 (2004).

2. Michaely, P., Tomchick, D. R., Machius, M. \& Anderson, R. G. Crystal structure of a 12 ANK repeat stack from human ankyrinR. EMBO J. 21, 6387-6396 (2002).

3. Howard, J. \& Bechstedt, S. Hypothesis: A helix of ankyrin repeats of the NOMPC-TRP ion channel is the gating spring of mechanoreceptors. Curr. Biol. $14,224-226$ (2004)

4. Corey, D. P. et al. TRPA1 is a candidate for the mechanosensitive transduction channel of vertebrate hair cells. Nature 432, 723-730 (2004).

5. Sotomayor, M., Corey, D. P. \& Schulten, K. In search of the hair-cell gating spring: Elastic properties of ankyrin and cadherin repeats. Structure 13, 669-682 (2005).

6. Schmid, E. L., Keller, T. A., Dienes, Z. \& Vogel, H. Reversible oriented surface immobilization of functional proteins on oxide surfaces. Anal. Chem. 69, 1979-1985 (1997).

7. Schmitt, L., Ludwig, M., Gaub, H. E. \& Tampe, R. A metal-chelating microscopy tip as a new toolbox for single-molecule experiments by atomic force microscopy. Biophys. J. 78, 3275-3285 (2000).

8. Rief, M., Gautel, M., Oesterhelt, F., Fernandez, J. M. \& Gaub, H. E. Reversible unfolding of individual titin immunoglobulin domains by AFM. Science 276, 1109-1112 (1997).

9. Oberhauser, A. F., Marszalek, P. E., Erickson, H. P. \& Fernandez, J. M. The molecular elasticity of the extracellular matrix protein tenascin. Nature 393, 181-185 (1998).

10. Marszalek, P. E. et al. Mechanical unfolding intermediates in titin modules. Nature 402, 100-103 (1999).

11. Fisher, T. E., Marszalek, P. E. \& Fernandez, J. M. Stretching single molecules into novel conformations using the atomic force microscope. Nature Struct. Biol. 7, 719-724 (2000).

12. $\mathrm{Li}, \mathrm{H}$. B. et al. Reverse engineering of the giant muscle protein titin. Nature 418 , 998-1002 (2002)
13. Williams, P. M. et al. Hidden complexity in the mechanical properties of titin. Nature 422, 446-449 (2003)

14. Oberhauser, A. F., Baddilla-Fernandez, C., Carrion-Vazquez, M. \& Fernandez, J. M. The mechanical hierarchies of fibronectin observed with single-molecule AFM. J. Mol. Biol. 319, 433-477 (2002).

15. Fernandez, J. M. \& Li, H. B. Force-clamp spectroscopy monitors the folding trajectory of a single protein. Science 303, 1674-1678 (2004).

16. Rief, M., Pascual, J., Saraste, M. \& Gaub, H. E. Single molecule force spectroscopy of spectrin repeats: Low unfolding forces in helix bundles. J. Mol. Biol. 286, 553-561 (1999).

17. Schwaiger, I., Schleicher, M., Noegel, A. \& Rief, M. The folding pathway of a fast-folding immunoglobulin domain revealed by single-molecule mechanical experiments. EMBO J. 6, 46-51 (2005).

18. Bustamante, C., Marko, J. F., Siggia, E. D. \& Smith, S. Entropic elasticity of $\lambda$-phage DNA. Science 265, 1599-1600 (1994).

19. Corey, D. P. \& Sotomayor, M. Tightrope act. Nature 428, 901-903 (2004).

20. Ashmore, J. Channel at the hair's end. Nature 432, 685-686 (2004).

21. Howard, J. \& Hudspeth, A. J. Compliance of the hair bundle associated with gating of mechanoelectrical transduction channels in the bullfrog's saccular hair cell. Neuron 1, 189-199 (1988).

22. Best, R. B. et al. Force mode atomic force microscopy as a tool for protein folding studies. Anal. Chim. Acta 479, 87-105 (2003).

23. Tang, K. S., Fersht, A. R. \& Itzhaki, L. S. Sequential unfolding of ankyrin repeats in tumour suppressor p16. Structure 11, 67-73 (2003).

24. Mello, C. C. \& Barrick, D. An experimentally determined protein folding energy landscape. Proc. Natl Acad. Sci. USA 101, 14102-14107 (2004).

25. Schwaiger, I., Sattler, C., Hostetter, D. R. \& Rief, M. The myosin coiled-coil is a truly elastic protein structure. Nature Mater. 1, 232-235 (2002).

26. Cecconi, C., Shank, E. A., Bustamante, C.\& Marqusee, S. Direct observation of the three-state folding of a single protein molecule. Science 309, 2057-2060 (2005).

27. Bennett, V. \& Baines, A. J. Spectrin and ankyrin-based pathway: Metazoan inventions for integrating cells into tissues. Physiol. Rev. 81, 1353-1392 (2001).

28. Florin, E. L. et al. Sensing specific molecular interactions with the atomic force microscope. Biosens. Biolelectron. 10, 895-901 (1995).

Supplementary Information is linked to the online version of the paper at www.nature.com/nature.

Acknowledgements We thank P. Snyder and E. Toone for help with the functionalization of glass coverslips, and B. Sunda and D. Corey for discussions. This work was supported by Duke University funds and grants from the National Science Foundation (to P.E.M.) and the Howard Hughes Medical Institute (to V.B.)

Author Information Reprints and permissions information is available at npg.nature.com/reprintsandpermissions. The authors declare no competing financial interests. Correspondence and requests for materials should be addressed to P.E.M. (pemar@duke.edu) or V.B. (benne012@mc.duke.edu). 